\title{
Speech Emotion Recognition Using Multi-granularity Feature Fusion Through Auditory Cognitive Mechanism
}

\author{
Cong $\mathrm{Xu}^{1(\bowtie)}$, Haifeng $\mathrm{Li}^{1,2(\bowtie)}$, Hongjian $\mathrm{Bo}^{2(\bowtie)}$, and Lin $\mathrm{Ma}^{1,2(\bowtie)}$ \\ ${ }^{1}$ Harbin Institute of Technology, 92, West Dazhi Street, Nan Gang District, \\ Harbin, People's Republic of China \\ xucong08@163.com, lihaifeng@hit.edu.cn, \\ malin_li@lhit.edu.cn \\ 2 Shen Zhen Academy of Aerospace Technology, 6, Keji South 10th Road, \\ Nan Shan District, Shen Zhen, People's Republic of China \\ bosaat@163.com
}

\begin{abstract}
In this paper, we focus on the problems of single granularity in feature extraction, loss of temporal information and inefficient use of frame features in discrete speech emotion recognition. Firstly, preliminary cognitive mechanism of auditory emotion is explored through cognitive experiments, and then a multi-granularity fusion feature extraction method inspired by the mechanism for discrete emotional speech signals is proposed. The method can extract 3 different granularity features, including short-term dynamic features of frame granularity, dynamic features of segment granularity and long-term static features of global granularity. Finally, we use the LSTM network model to classify emotions according to the long-term and short-term characteristics of the fusion features. We implement experiment on the discrete emotion datasets of CHEAVD (CASIA Chinese Emotional Audio-Visual Database) released by the Institute of automation, China Research Academy of Sciences, and achieved improvement in recognition rate, increasing the MAP by $6.48 \%$.
\end{abstract}

Keywords: Speech emotion recognition - Auditory cognitive mechanism • Multi-granularity feature fusion $\cdot$ CNN-LSTM

\section{Introduction}

Speech emotion recognition is to judge the current emotional state by analyzing and processing the audio signals of human speech. The stimulation of the emotions is related to the cognitive response in the brain after the speech signal is acquired. Thus, the analysis and the estimation of speech emotion based on the auditory cognitive mechanism is the focal points and difficulty in the research of intelligent humancomputer interaction, and is the important foundation for the realization of machine hearing $[1,2]$. Because of the non-stationary and time-series characteristics of speech signals, the analysis and extraction of emotional information contained in speech signals has become the focus of speech emotion recognition task, and it is also a hotspot of current research. 
Researchers usually use two kinds of acoustic features for the speech emotion recognition. One is the global statistical features based on sentences, including prosodic features [3-5], power spectrum features [6, 7] and voice quality features [8, 9]. Seppänen et al. [10] used 43-dimensional global prosodic features related to fundamental frequency, energy and duration to recognize the emotion of Finnish speech, and achieved $60 \%$ recognition rate in the case of speaker-independent. Li et al. [11] used frequency perturbation and amplitude perturbation as voice quality features to recognize speaker-independent emotions in SUAS database, increasing the recognition rate from $65.5 \%$ to $69.1 \%$. He et al. [12] extracted the area under the envelope of glottic waveform energy spectrum and the area under the envelope of speech signal energy spectrum as voice quality features, and achieved good recognition performance. Sanchez et al. [13] achieved outstanding results in the fusion of different types of features. They applied 90-dimensional global statistical features, such as fundamental frequency, energy, resonance peak and spectral tilt, to detect the emotion of depression in WCGS database [14], and achieved $81.3 \%$ recognition rate. Haifeng et al. [15] realized the recognition of Activation, Dominance and Valence in dimensional speech emotion recognition by fusing different global granularity features. The other one is time sequential features based on speech frames. As the emotional speech features are unequal in length and unsteady in the audio signal [16, 17], Li et al. [18] proposed vowel temporal features based on global features and implemented four kinds of emotion recognition experiments, which achieved good recognition results.

In these studies, speech emotion features are mostly extracted by frame, but use the global static feature of a sentence or longer speech segment in the progress of recognition [19]. This kind of feature cannot reflect the dynamic change of speech emotion during the speaker's speaking. In order to extract better features that can describe the dynamic changes of emotion in speech, researchers have started exploring the emotion features contained in audio signals from the perspective of auditory cognition.

Thus, in this paper, we implemented a speech emotion cognitive experiment to explore the auditory cognitive mechanism of how the brain process the speech during the recognition of emotions. Inspired by the cognitive mechanism, we proposed a multi-granularity dynamic and static fusion feature and the method to extract it. This fusion feature can avoid the problem of unequal length of the dynamic features, and retains as much emotion information as possible at the same time. The framework of the work is shown in Fig. 1. We first calculated the prosodic features, spectrum features and voice quality features from the frames. Then the static global granularity features of the whole sentence are obtained by statistical calculation. At the same time, we use the Gauss window to convolute the adjacent frame features in time series, and get the multi-granularity time-varying dynamic features, which can not only depict the speaker's overall speech features, but also describe the change of speech emotion features over time.

For the classification part, we use Long-Short Term Memory (LSTM) network model. LSTM model can effectively model time series and make full use of time series information in speech. On the other hand, LSTM's long-term and short-term memory mechanism enables the network to selectively remember and recognize features at different time points. And at last, LSTM network model can deal with variable length input effectively, so as to solve the problem that different utterance has different number of frames. 


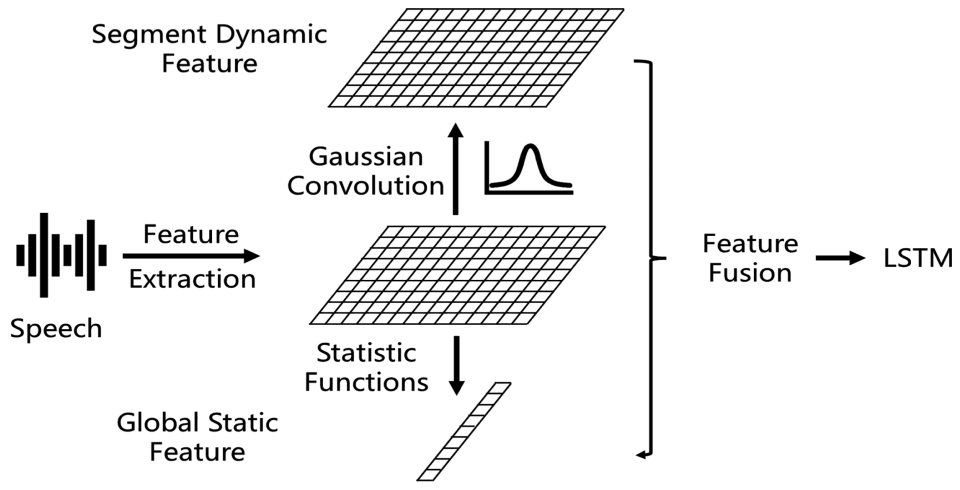

Fig. 1. The framework of speech emotion recognition

\section{Exploration of Auditory Cognitive Mechanism}

And that of human beings. A man can easily feel the speaker's emotions from a speech, even if he does not understand the language in the speech. This indicates semantics is not a key factor in the emotion recognition. Compared with human beings, the recognition rate of current speech emotion recognition methods is still at a relatively low level. Therefore, we intend to explore the mechanism of human brain in speech emotion recognition by analyzing the cognitive activities when hearing audios with different emotions, and use the mechanism to optimize the speech emotion features.

\subsection{Exploratory Experiment on Auditory Cognitive Mechanism}

There are many kinds of sound in this world, and different sound, such as the speech, music, noise etc., can evoke different emotions. In this work, we select sounds with low auditory attribute complexity and semantic complexity from 3 open database of emotional sounds: (1) IADS2 (2007); (2) Montreal Affective Voices (MAV, 2008); (3) Musical Emotional Bust (MEB, 2013). The sounds include living sound, non-living sound, wind sound, water sound, other natural environment sounds, and piano solos in different modes representing different emotions. With all these sound, we conducted a cognitive experiment of auditory emotion. We asked subjects to listen to different kinds of sounds, and recorded the EEG data of the subjects in the experiment using 64 electrodes, sampling with $1000 \mathrm{~Hz}$. And then analyze the cognitive mechanism during the process of emotional change.

The subjects in the experiment are consist of 15 health college students aged between 19 and 22, including 7 women. And all subjects were right-handed. For each subject, we conduct 16 trials, the timing scheme of each trial is as follows:

(1) Record 15 s continuous EEG signal of the subject;

(2) Play a clip of one of the sounds, human voices, or music;

(3) Record another 15 s continuous EEG signal of the subject;

(4) Self assessment and scoring of the subject's emotional state. 


\subsection{Result and Analysis}

We analyze the EEG signals collected under different emotions by time-related potential (ERP), and the results were shown in Fig. 2. Firstly, we compared the ERP of living sound and non-living sound, significant differences in N1-P2 components can be found at FZ, CZ and other electrodes. And Auditory-object Specific Response (ASR) components related to object category can be found at $350 \mathrm{~ms}$, which is consistent with the results of Levy [20], Murray [21] and other researchers. This proves that the experiment is correctly conducted. Then we compared the ERP of different emotion sound. Similarly, significant differences in N1-P2 components can be found at FZ, CZ and other electrodes, while no ASR component can be found at $350 \mathrm{~ms}$, but new emotional components can be found around $400 \mathrm{~ms}$, which we call ESR (Emotion Specific Response). This indicates that the earliest response of human brain to emotion is about $150 \mathrm{~ms}$ in N1-P2 auditory components, and the most significant difference in ESR components occurs at $450 \mathrm{~ms}$. In addition, the time of ESR component of emotion is about $100 \mathrm{~ms}$ later than that of ASR component of object category, which indicates that emotion has a higher level of information processing.
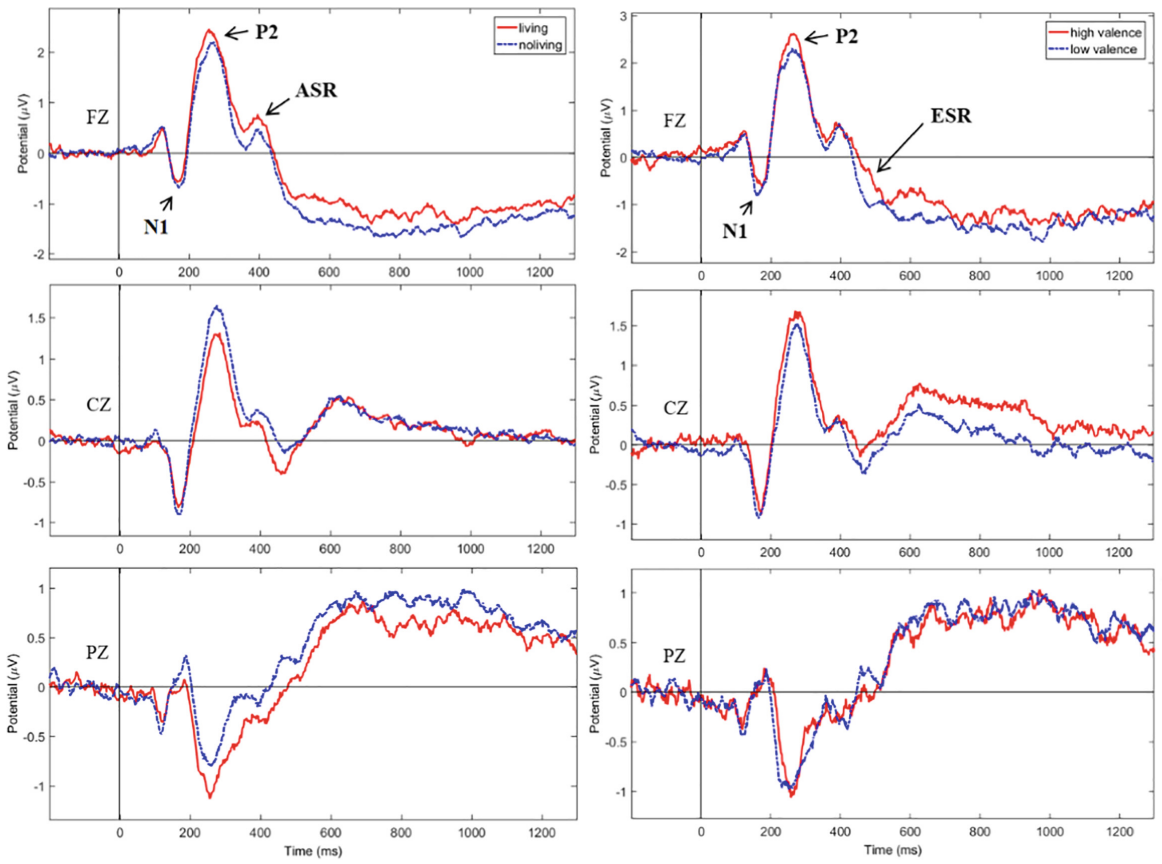

Fig. 2. ERP analysis of sound evoked emotions. ERP of living and non-living sounds (left); ERP of high and low valence sounds (right). 
We also plotted the brain topographic map of EEG signals in timing series, as shown in Fig. 3. We can see that the activated brain regions of different emotion changed at $350-450 \mathrm{~ms}$. That is different neural pathways is using for processing emotional information.

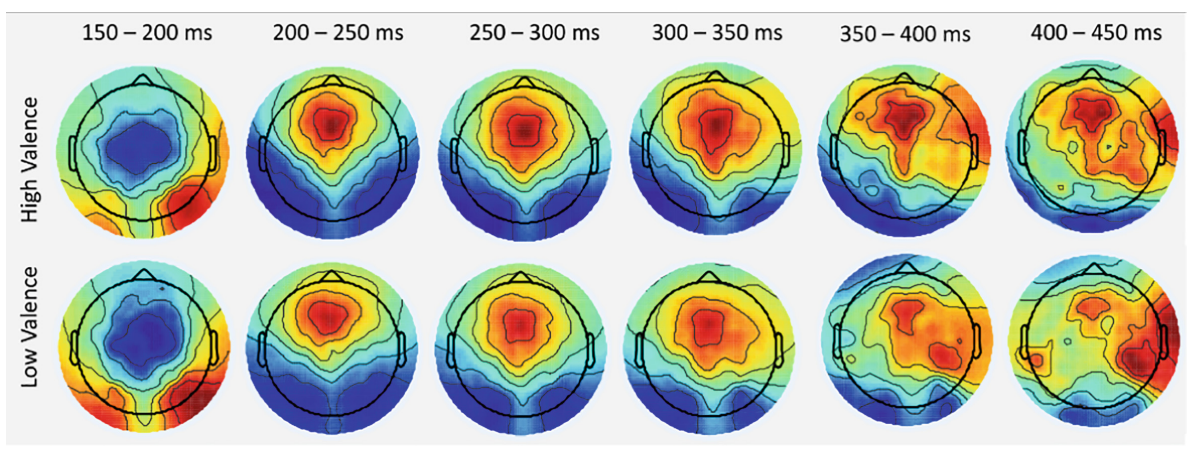

Fig. 3. Time series brain topographic maps of sound evoked emotions.

\subsection{Enlightenment to Speech Emotion Features}

Through the aforementioned analysis of EEG data of human brain in emotional cognitive activities, we have obtained some preliminary mechanism of emotional cognitive. These mechanism suggest that we should pay attention to the following points in speech emotion recognition:

Emotion is Based on Acoustic Features but More Than Acoustic Features. It takes more time for brain to respond to the living sound than to the non-living sound, and emotional components of the brain appear between $350 \mathrm{~ms}$ and $450 \mathrm{~ms}$. This indicates that the brain obviously undergoes more advanced information processing mechanism when hearing a human speech. The evocation of emotion in human brain is not a simple stimulus-response process, but involves in the arrangement and combination of acoustic features. From this we can infer that emotional information is based on acoustic features, but more than acoustic features. It is not necessarily effective to extract features from simple acoustic attributes of speech signals.

The Response Time Varies from Different Emotion. The response time of the brain to different emotions is slightly different, therefore, multi-granularity features will play an important role in speech emotion and even sequence signals, facilitating the further extraction of emotional information. Moreover, the response time of human brain to emotion information is $350-450 \mathrm{~ms}$, which provides a theoretical basis for the size of the granularity. In the later experiments, we set the length of the window to extract segment granularity feature to $350 \mathrm{~ms}$. 


\section{Extraction Method for Multi-Granularity Fusion Feature}

In view of the above-mentioned enlightenment, we propose a multi-granularity dynamic and static fusion feature extraction method. We divide features into three granularities: frame, segment and global. The first two correspond to dynamic features, and the last correspond to static features. In this way, the temporal information in audio is preserved, and the ability of feature expression is improved. The definition of multigranularity dynamic-static fusion features is shown in Fig. 4.

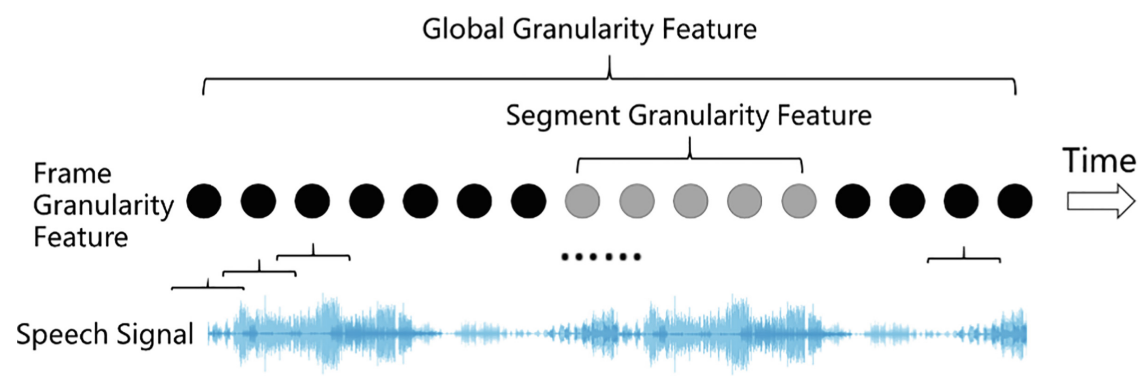

Fig. 4. The definition of multi-granularity dynamic-static fusion feature.

\subsection{Dynamic Feature Extraction Method}

Frame Granularity Dynamic Feature. Frame feature is the smallest granularity in speech signal analysis and processing. It represents the short-term features of speech. The frame feature sequence obtained from an utterance also retains most kinds of information about how the signal varies with time.

In this work, we divided the continuous speech signal into several approximate stationary speech frames. The frame length was $25 \mathrm{~ms}$, the frame shift was $10 \mathrm{~ms}$, window function was Hamming window. We extract 65-dimensional features including fundamental frequency, short-term energy, short-term average energy, zerocrossing rate, average amplitude difference, resonance peak and MFCC. The specific features are shown in Table 2.

Segment Granularity Dynamic Feature. When the human brain processes an utterance and extracts information, it does not recognize it one by one at the smallest granularity. It needs an advanced processing in a bigger granularity to extract emotional information. Therefore, based on the dynamic feature of frame granularity, we propose a dynamic feature extraction of segment granularity with $350 \mathrm{~ms}$ window length.

Segment feature is obtained by convoluting the frame features within a certain window length using a convolution function. This makes segment feature be able to reflect the temporal relationship between consecutive frames, which means that the dynamic changing process is depicted. We use $x_{t}=\left(a_{(t, 1)}, a_{(t, 2)}, \ldots, a_{(t, P)}\right)$ to denote the frame feature vector at time $t$, where $P$ is the dimension of the frame feature. An 
utterance of length $T$ can be expressed by a frame feature matrix consist of frame feature vectors as follows:

$$
X_{T}^{T}=\left(x_{1}, x_{2}, \ldots, x_{T}\right)
$$

For each frame feature matrix of $P \times T$ size, we use pre-defined segment length $L$, here is $350 \mathrm{~ms}$, and corresponding convolution function group $K(M, T)$ to convolute it, where $M$ is the number of convolution functions in the convolution function group. Then the segment feature matrix $S_{M \times T}$ is obtained:

$$
S_{(m, t)}=K_{(m, t)} *\left(x_{t-L+1}, x_{t-L+2}, \ldots, x_{t}\right)^{T}
$$

As the emotion in an utterance usually rises at first and then degrade. The middle part usually carries the most emotional information, while the two ends are relatively less. So we use different Gaussian function $G(m, t)$, as shown in Fig. 5, as the convolution function to convolute frame features in different positions in the segment window.

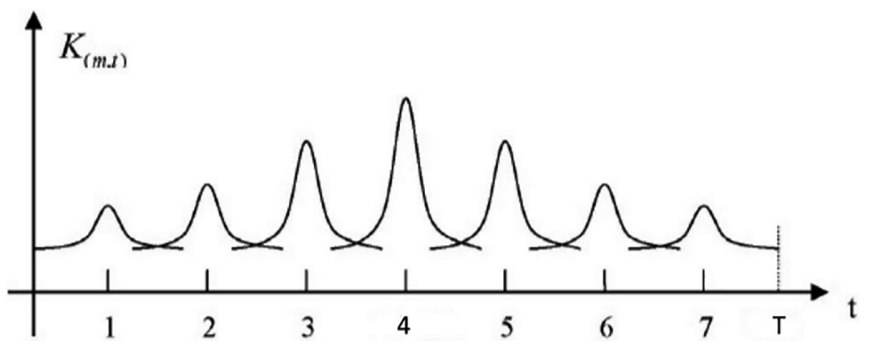

Fig. 5. Gaussian function group.

The Gaussian function we used can be expressed as:

$$
G_{(m, t)}=\frac{1}{\sqrt{2 \pi} \sigma_{m}} e^{-\frac{\left(t-m T_{D}\right)^{2}}{2 \sigma_{m}^{2}}}
$$

Where $\sigma_{m}$ can be computed through:

$$
\sigma_{m}^{2}=\sigma_{\frac{M+1}{2}}^{2}\left(m-\frac{M+1}{2}\right)^{2}
$$

After the above steps, we transform the frame feature matrix of $P \times T$ size into segment feature matrix of $M \times T$ size. Since the dimension of time is not changed, segment feature still retains the timing characteristics of the original utterance and the advanced information contained in it. 


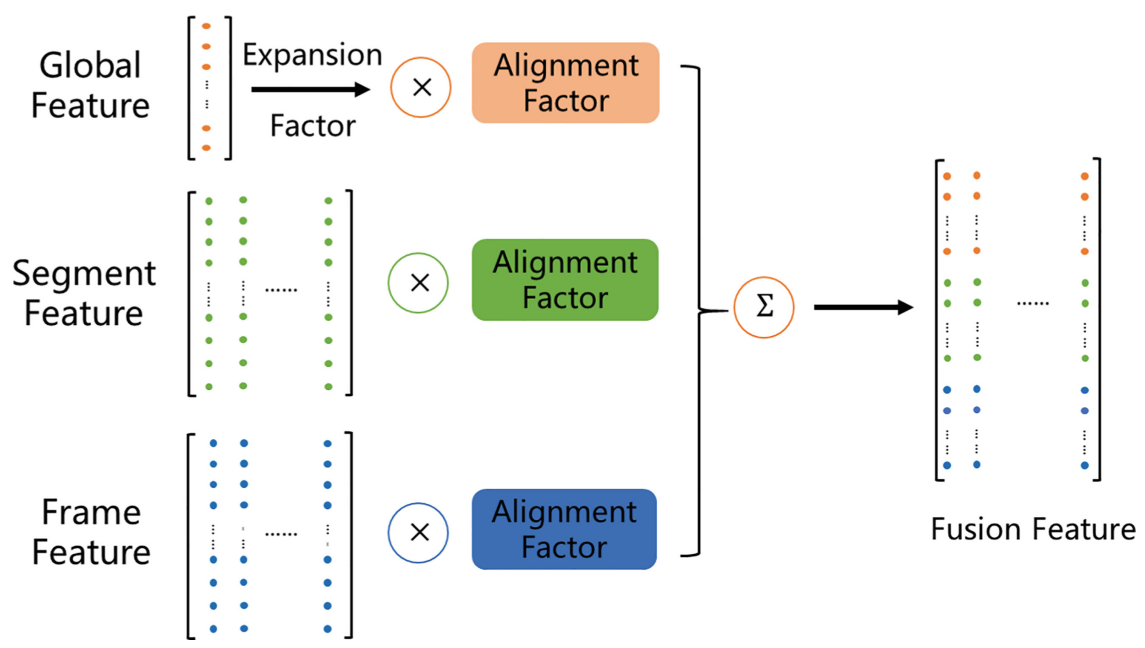

Fig. 6. Process of multi-granularity dynamic-static features fusion.

\subsection{Static Feature Extraction Method}

For static features, we use the current mainstream feature extraction method, which is to obtain global static features by calculating the corresponding frame feature statistics on the entire utterance. The statistics we calculate here include the maximum value, the minimum value, the average value, the variance, etc.

\subsection{Fusion Method of Multi-Granularity Dynamic-Static Features}

When the three features are fused, since the global static feature is calculated by the frame feature through the statistical function, then the frame feature with dimension $\mathrm{P}$ will be transformed into global statistical feature with dimension $P * R$, after the global statistical calculation is performed using $R$ statistical functions. That means the dimension of global feature will be $\mathrm{R}$ times that of frame feature, which makes the global part of the fused feature account for the vast majority of the total. But in fact, dynamic information as time changes, corresponding to the frame feature and the segment feature, is relatively more important.

Therefore, in the feature fusion, we first use the PCA algorithm to select the feature of the calculated global static feature, keep the first $P_{g}$ dimension, so that the dimension ratio of the dynamic feature and the static feature are balanced. And finally, the global static feature $\boldsymbol{G}_{P_{g}}$ becomes a vector of $P_{g}$ dimension.

We set the alignment factor matrices $A_{x}, A_{s}, A_{g}$ for the frame feature matrix $X_{P \times T}$, the segment feature matrix $S_{M \times T}$, and the global feature vector $\boldsymbol{G}_{P_{g}}$, respectively. Then the fusion feature matrix $\mathrm{F}$ can be computed through the following equation: 


$$
F_{\left(P+M+P_{g}\right) \times T}=A_{x} X_{P \times T}+A_{s} S_{M \times T}+A_{g} \boldsymbol{G}_{P_{g}} \boldsymbol{B}_{g}
$$

Where $\boldsymbol{B}_{g}$ is a T-dimensional vector consisting of T ones, we call it global feature vector expansion factor. The alignment factor matrices are set as follows:

$$
\begin{aligned}
A_{x} & =\left(\begin{array}{c}
\mathbf{0} \\
\mathbf{0} \\
I_{p}
\end{array}\right)_{\left(P+M+P_{g}\right) \times T} \\
A_{s} & =\left(\begin{array}{c}
\mathbf{0} \\
I_{S} \\
\mathbf{0}
\end{array}\right)_{\left(P+M+P_{g}\right) \times T} \\
A_{g} & =\left(\begin{array}{c}
I_{P_{g}} \\
\mathbf{0} \\
\mathbf{0}
\end{array}\right)_{\left(P+M+P_{g}\right) \times T}
\end{aligned}
$$

Where $I$ is unit array.

Thus each column in matrix $F$ is a fused multi-granularity feature consisting of 3 parts: dynamic frame granularity features, segment granularity dynamic features obtained by convolution on frame features, and global statistical static feature based on all frames in the utterance. The feature fusion process is shown in Fig. 6.

\section{Speech Emotion Recognition Experiment}

\subsection{Dataset}

We used the CHEAVD (Chinese Natural Emotional Audio-Visual Database) dataset [22], which is constructed by the team of Institute of Automation, Chinese Academy of Sciences. The data are from the audio and video clips intercepted in the movies and TV shows. And in this experiment, we only use the audio clips data for the speech emotion recognition. There are total of 8 kinds of emotion in the dataset, and the distribution of the sample size for each emotion is shown in Table 1 below.

From the table we can see that, the sample numbers of different emotion are not balance. "Neutral" has 815 samples which is the most, while "Disgust" only has 50 . Moreover, the data is collected from many different scenarios, including movies, TV plays, talk shows, etc. So it is very close to the emotional expression in real life. This makes it difficult and challenging to recognize emotion on the dataset.

\subsection{Feature Set}

We extract features on the original audio in CHEAVD using OpenSmile [23], an open source tool. We refer to the feature set in The INTERSPEECH 2016 Computational Paralinguistic Challenge [24], and select 65 of them as our frame feature, as is listed in Table 2. 

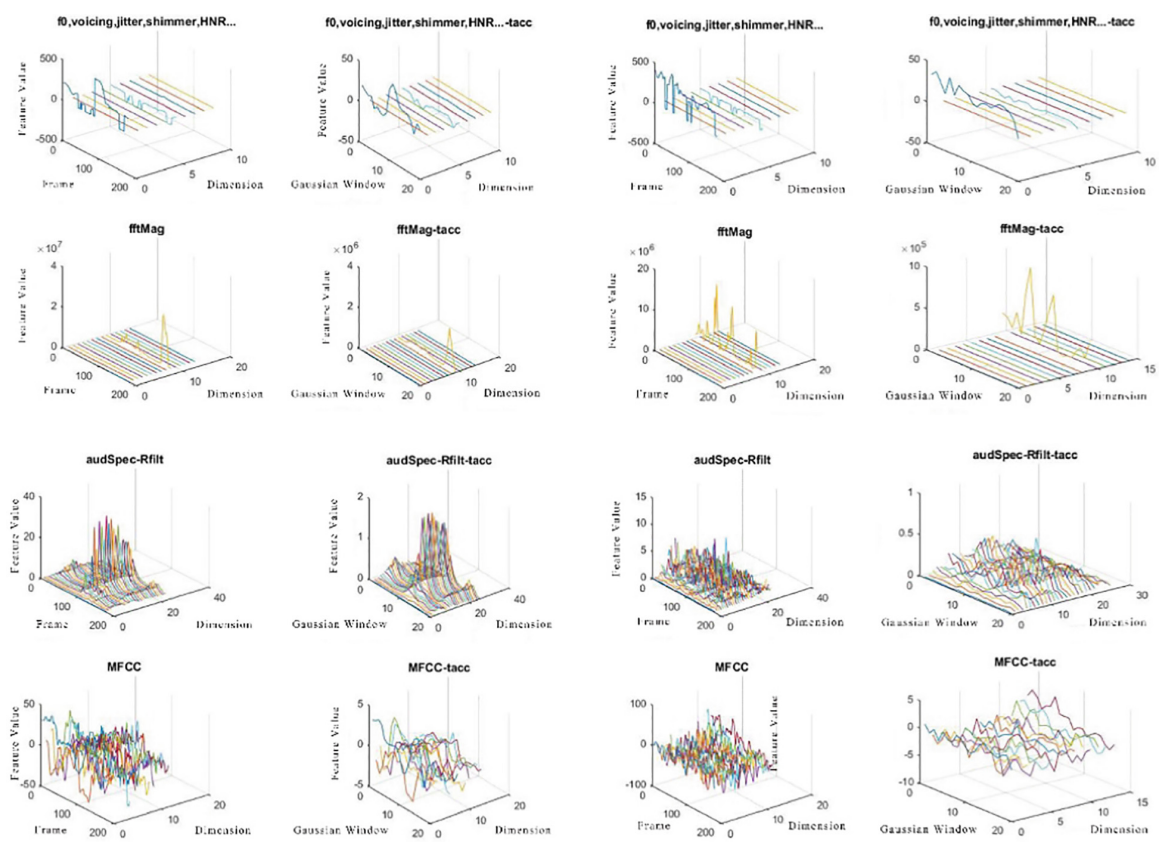

(a)

(b)

Fig. 7. Comparison of frame features and segment dynamic features. (a) features of emotion 'surprise': frame feature (left), segment feature (right); (b) features of emotion 'angry': frame feature (left), segment feature (right).

Table 1. Sample distribution of eight emotion classes.

\begin{tabular}{l|r|r|r}
\hline Emotion & Train sample number & Test sample number & Total \\
\hline Neutral & 725 & 90 & 815 \\
\hline Angry & 399 & 49 & 448 \\
\hline Happy & 307 & 38 & 345 \\
\hline Sad & 256 & 31 & 387 \\
\hline Worried & 93 & 11 & 104 \\
\hline Anxious & 89 & 11 & 100 \\
\hline Surprise & 67 & 8 & 75 \\
\hline Disgust & 45 & 5 & 50 \\
\hline Total & 1981 & 243 & 2224 \\
\hline
\end{tabular}

After getting the frame features, we calculate the segment features using the method mentioned above. Figure 7 show the feature comparison of the emotion "surprise" and "angry" respectively. In the figure, the left are the frame features, and the right are the segment features. And we can see that the curve of segmental granularity dynamic features obtained by convolution calculation is smoother and more regular. 
Table 2. Frame feature set list.

\begin{tabular}{l|l|c}
\hline Feature type & Feature abbr. & Dimension \\
\hline \multirow{3}{*}{ Prosodic features } & Fofinal & 1 \\
\cline { 2 - 3 } & voicingFinalUnclipped & 1 \\
\cline { 2 - 3 } & Zcr & 1 \\
\hline Voice quality features & MFCC & 14 \\
\cline { 2 - 3 } & pcm RMSenergy & 1 \\
\cline { 2 - 3 } & audSpec Rfilt & 28 \\
\cline { 2 - 3 } & jitterLocal & 15 \\
\cline { 2 - 3 } & jitterDDP & 1 \\
\cline { 2 - 3 } & shimmerLocal & 1 \\
\cline { 2 - 3 } & logHNR & 1 \\
\hline
\end{tabular}

In terms of global statistical static features, we selected 16 statistic functions as shown in Table 3 . We would get $65 * 16=1040$ dimensional global feature after applying 16 statistic function on 65-dimensional frame feature. To avoid the dimension imbalance of three different granularity, we use the PCA algorithm to reduce the global features dimension, and select the first 65 dimensions as the final global static feature.

Table 3. Statistic functions list.

\begin{tabular}{l|l}
\hline Type & Statistics \\
\hline Extremum & max/min, location of max/min \\
\hline Mean value & geometric mean, arithmetic mean, square mean \\
\hline Moment & variance, standard deviation, skewness, plainness \\
\hline Regression coefficient & correlation coefficient of quadratic regression \\
\hline Quantile & Quartile(0.25/0.5/0.75/0.9) \\
\hline
\end{tabular}

\subsection{Experiment and Result Analysis}

We conduct 4 different experiments on the dataset, using different features and classifiers. We use 88-dimensional global feature, which is the feature used in the baseline method, to compare the effects of different classifiers. We also only use 65-dimensional frame feature, which is the same as the frame granularity feature in the fusion feature, to verify the role of fusion features. For the classifiers, we use single layer LSTM and SVM. In LSTM, the number of hidden layer nodes is 128 , the activation function is tanh function, and the output layer uses the softmax function.

Table 4 shows all the experimental results. The first line and the sixth line are the baseline method and winner's method for 2016 Multi-mode Speech Emotion Recognition Competition on the dataset. The accuracy and the macro average precision 
(MAP) are calculated. MAP is widely used to measure the classification effect of classifier. Its calculation formula is as follows.

Table 4. Comparison of experiment result.

\begin{tabular}{l|l|l|l}
\hline No. & Features - Method & Acc on Test set & MAP(\%) \\
\hline 1 & 88-dimensional global feature - decision tree & $41.98 \%$ & 30.02 \\
\hline 2 & 88-dimensional global feature - LSTM & $41.56 \%$ & 31.56 \\
\hline 3 & 88-dimensional global feature - SVM & $40.9 \%$ & 28.94 \\
\hline 4 & 65-dimensional frame feature - LSTM & $41.98 \%$ & 33.24 \\
\hline 5 & Multi-granularity fusion feature - LSTM & $44.26 \%$ & 36.5 \\
\hline 6 & DFFN based on GC & $45.24 \%$ & 20.81 \\
\hline
\end{tabular}

$$
M A P=\frac{1}{S} \times \sum_{i=1}^{s} \frac{T P_{i}}{T P_{i}+F P_{i}}
$$

Where $T P_{i}$ is the number of correct predict in class $i, F P_{i}$ is the number of the samples of other classes identified as the class $i$.

From the table we can see that the method using multi-granularity fusion features has improved the recognition rate compared to the baseline method. Meanwhile, the effect of using fusion feature is better than using frame features alone or global features alone. This shows that multi-granularity feature extraction is helpful to improve the performance of the accuracy of the emotion recognition. On the other hand, the recognition result of using global features alone is lower than that of using frame features alone, which shows that the performance of frame features with more temporal information is better than that of global features in speech signals. We also compared the result with the method of the winner in the competition on the dataset [25]. In this method, the data are classified by sex first, and then uses deep feedforward neural network for classification. We can see from the results that although we have not classified the gender, our performance are still very close to its performance, and have achieved better results on the MAP value. Besides, all the above methods are better than traditional global statistical features and SVM classifiers.

We also calculated ROC curves for eight emotion classes, as shown in Fig. 7. The average area under the ROC curve (AUC) of the eight emotions reach 0.69, and the overall trend of the ROC curve of the eight emotions in the graph shows that the classifier using the multi-granularity dynamic-static fusion features has better performance, even for the emotion that is relatively difficult to recognize, such as 'anxious' and 'surprise' (Fig. 8). 


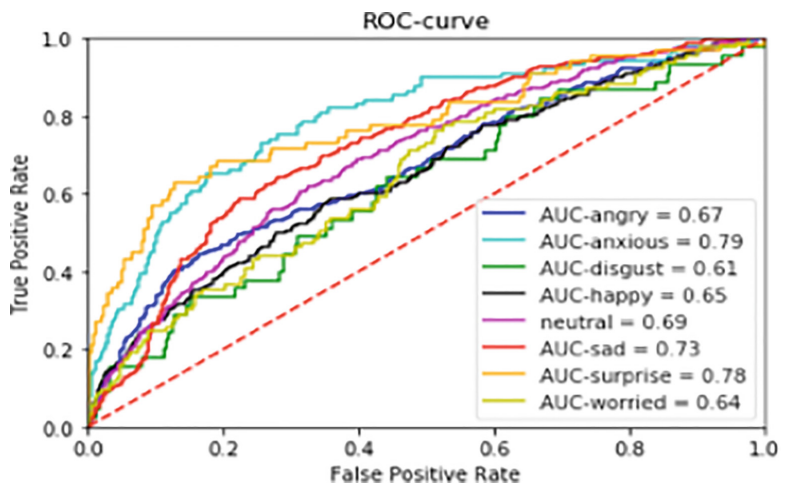

Fig. 8. ROC curve of 8 emotions.

\section{Conclusion}

In this paper, we aim at the problem of insufficient dynamic information processing mechanism in speech emotion recognition, and explores the auditory cognitive mechanism of speech emotion recognition by designing speech emotion recognition cognitive experiment. Based on the mechanism we get, a multi-granularity dynamic and static fusion feature extraction method is proposed. The frame feature, segment feature and global feature are extracted and fused to make full use of the frame feature and the temporal information contained in it. Meanwhile, the LSTM model is used to classify and recognize the long and short temporal information. Experiments show that multi-granularity fusion features can effectively improve the effect of speech emotion recognition. Finally, considering that there is no structural optimization of the LSTM network in this paper, there should be room for improvement in the recognition effect.

Acknowledgements. Our thanks to Gao Chang for the tremendous help provided in the experiment and supports from the National Key Research and Development Program of China (2018YFC0806800), National Natural Science Foundation of China (61671187), Shenzhen Foundational Research Funding (JCYJ20180507183608379, JCYJ20150929143955341), Shenzhen Key Laboratory of Innovation Environment Project (ZDSYS201707311437102), Open Funding of MOE-Microsoft Key Laboratory of Natural Language Processing and Speech (HIT. KLOF.20150xx, HIT.KLOF.20160xx), China Postdoctoral Science Foundation (2017M611187).

\section{References}

1. Ortony, A., Clore, G.L., Collins, A.: The Cognitive Structure of Emotions. Cambridge Univ Pr, Cambridge (1990)

2. Scherer, K.R.: Vocal communication of emotion: a review of research paradigms. Speech Commun. 40(1), 227-256 (2003)

3. Arias, J.P.: Shape-based modeling of the fundamental frequency contour for emotion detection in speech. Comput. Speech Lang. 28(1), 278-294 (2014) 
4. Zao, L.: Time-Frequency feature and AMS-GMM mask for acoustic emotion classification. IEEE Signal Process. Lett. 21(5), 620-624 (2014)

5. Mencatini, A., Martineli, E., Costantini, G., et al.: Speech emotion recognition using amplitude modulation parameters and a combined feature selection procedure. Knowl-Based Syst. 63, 68-81 (2014)

6. Huang, Y., Zhang, G., Li, Y., Wu, A.: Improved emotion recognition with novel taskoriented wavelet packet features. In: Huang, D.-S., Bevilacqua, V., Premaratne, P. (eds.) ICIC 2014. LNCS, vol. 8588, pp. 706-714. Springer, Cham (2014). https://doi.org/10.1007/ 978-3-319-09333-8_77

7. Ziolko, M., Jaciow, P., Igras, M.: Combination of Fourier and wavelet transformations for detection of speech emotions. In: 7th International Conference on Human System Interactions (HSI), pp. 49-54. IEEE (2014)

8. Idris, I., Salam, M.S.H.: Emotion detection with hybrid voice quality and prosodic features using neural network. In: 2014 Fourth World Congress on Information and Communication Technologies (WICT), pp. 205-210. IEEE (2014)

9. Kachele, M., Zharkov, D., Meudt, S., et al.: Prosodic, spectral and voice quality features selection using a long-term stopping criterion for audio-based emotion recognition. In: 2nd International Conference on Pattern Recognition (ICPR), pp. 803-808. IEEE (2014)

10. Seppänen, T., Väyrynen, E., Toivanen, J.: Prosody-based classification of emotions in spoken finnish. In: Proceedings the 2003 European Conference on Speech Communication and Technology. EUROSPEECH, pp. 717-720. ISCA, Geneva (2003)

11. Li, X., Tao, J., Johnson, M.T., et al.: Stress and emotion classification using jitter and shimmer features. In: Proceedings the 2007 IEEE International Conference on Acoustics, Speech, and Signal Processing (ICASSP), IV, pp. 1081-1084. IEEE, Honolulu (2007)

12. He, L., Lechm, M., Allen, N.: On the importance of glottal flow spectral energy for the recognition of emotions in speech. In: Proceedings the 2010 INTERSPEECH, pp. 23462349. ISCA, Chiba (2010)

13. Sanchez, M.H., Vergyri, D., Ferrer, L., et al.: Using prosodic and spectral features in detecting depression in elderly males. In: Proceedings the 2011 INTERSPEECH, pp. $3001-$ 3004. ISCA, Florence (2011)

14. Rosenman, R.H.: A predictive study of coronary heart disease: the western collaborative group study. JAMA 189(1), 15-22 (1964)

15. Jing, C., Haifeng, L., Lin, Ma., et al.: Multi-granularity feature fusion for dimensional speech emotion recognition. J. Sig. Process. 33(3), 374-382 (2017)

16. Cowie, R., Douglas-Cowie, E., Tsapatsoulis, N., et al.: Emotion recognition in human computer interaction. IEEE Signal Process. Mag. 18(1), 32-80 (2001)

17. Murray, I., Arnott, J.: Toward a simulation of emotion in synthetic speech: a review of the literature on human vocal emotion. J. Acoust. Soc. Am. 93(2), 1097-1108 (1993)

18. Li, Z., Zhiping, W., Wei, L., Cairong, Z., Zhenyang, W.: Speech emotional recognition using global and time sequence structure feature. Acta Automatica Sinica 30(3), 423-429 (2004)

19. Wenjing, H., Haifeng, L., Huabin, R., et al.: Review on speech emotion recognition. J. Softw. 25(1), 37-50 (2014)

20. Levy, D.A., Granot, R., Bentin, S.: Processing specificity for human voice stimuli: electrophysiological evidence. NeuroReport 12(12), 2653-2657 (2001)

21. Murray, M.M., Camen, C., Gonzalez Andino, S.L., et al.: Rapid brain discrimination of sounds of objects. J. Neurosci. 26(4), 1293-1302 (2006)

22. Li, Y., Tao, J., Schuller, B., Shan, S., Jiang, D., Jiam, J.: MEC 2016: the multimodal emotion recognition challenge of CCPR 2016. In: Chinese Conference on Pattern Recognition (CCPR), Chengdu (2016) 
23. Eyben, F., Weninger, F., Gross, F., Schuller, B.: Recent developments in openSMILE, the Munich open-source multimedia feature extractor. In: Proceedings of the 21st ACM International Conference on Multimedia, pp. 835-838 (2013)

24. Schuller, B., et al.: The INTERSPEECH 2016 computational paralinguistics challenge: Deception, sincerity \& native language. In: INTERSPEECH, pp. I-I. ISCA (2016)

25. Huang, Y., Hu, M., Yu, X., Wang, T., Yang, C.: Transfer learning of deep neural network for speech emotion recognition. In: Tan, T., Li, X., Chen, X., Zhou, J., Yang, J., Cheng, H. (eds.) CCPR 2016. CCIS, vol. 663, pp. 721-729. Springer, Singapore (2016). https://doi.org/ 10.1007/978-981-10-3005-5_59 